\title{
The Gaia-ESO Survey
}

\section{Mg-Al anti-correlation in iDR4 globular clusters ${ }^{\star} \star \star$}

\author{
E. Pancino ${ }^{1,3}$, D. Romano ${ }^{2}$, B. Tang ${ }^{4}$, G. Tautvaišiené ${ }^{5}$, A. R. Casey ${ }^{6}$, P. Gruyters ${ }^{7}$, D. Geisler ${ }^{4}$, I. San Roman ${ }^{8}$, \\ S. Randich ${ }^{1}$, E. J. Alfaro ${ }^{9}$, A. Bragaglia ${ }^{2}$, E. Flaccomio ${ }^{10}$, A. J. Korn ${ }^{11}$, A. Recio-Blanco ${ }^{12}$, R. Smiljanic ${ }^{13}$, \\ G. Carraro ${ }^{14}$, A. Bayo ${ }^{15}$, M. T. Costado ${ }^{9}$, F. Damiani ${ }^{10}$, P. Jofré6, 16, C. Lardo ${ }^{17}$, P. de Laverny ${ }^{12}$, L. Monaco ${ }^{18}$, \\ L. Morbidelli ${ }^{1}$, L. Sbordone ${ }^{19,20}$, S. G. Sousa ${ }^{21}$, and S. Villanova ${ }^{4}$
}

1 INAF-Osservatorio Astrofisico di Arcetri, Largo Enrico Fermi 5, 50125 Firenze, Italy e-mail: pancino@arcetri.inaf.it

2 INAF-Osservatorio Astronomico di Bologna, via Ranzani 1, 40127 Bologna, Italy

3 ASI Science Data Center, via del Politecnico snc, 01333 Roma, Italy

4 Departamento de Astronomía, Casilla 160-C, Universidad de Concepción, Concepción, Chile

5 Institute of Theoretical Physics and Astronomy, Vilnius University, Sauletekio av. 3, 10257 Vilnius, Lithuania

6 Institute of Astronomy, University of Cambridge, Madingley Road, Cambridge CB3 OHA, UK

7 Lund Observatory, Department of Astronomy and Theoretical Physics, Box 43, 22100 Lund, Sweden

8 Centro de Estudios de Física del Cosmos de Aragón (CEFCA), Plaza San Juan 1, 44001 Teruel, Spain

9 Instituto de Astrofísica de Andalucía-CSIC, Apdo. 3004, 18080 Granada, Spain

10 INAF-Osservatorio Astronomico di Palermo, Piazza del Parlamento 1, 90134 Palermo, Italy

11 Department of Physics and Astronomy, Uppsala University, Box 516, 75120 Uppsala, Sweden

12 Laboratoire Lagrange (UMR 7293), Université de Nice Sophia Antipolis, CNRS, Observatoire de la Côte d'Azur, CS 34229, 06304 Nice Cedex 4, France

13 Nicolaus Copernicus Astronomical Center, Polish Academy of Sciences, ul. Bartycka 18, 00-716 Warsaw, Poland

14 Dipartimento di Fisica e Astronomia, Università di Padova, Vicolo dell'Osservatorio 3, 35122 Padova, Italy

15 Instituto de Física y Astronomiía, Universidad de Valparaíso, Avda. Gran Bretaña 1111, Valparaiso, Chile

16 Núcleo de Astronomía, Facultad de Ingeniería, Universidad Diego Portales, Av. Ejercito 441, Santiago, Chile

17 Astrophysics Research Institute, Liverpool John Moores University, 146 Brownlow Hill, Liverpool L3 5RF, UK

18 Departamento de Ciencias Fisicas, Universidad Andres Bello, Fernandez Concha 700, Las Condes, Santiago, Chile

19 Millennium Institute of Astrophysics, Av. Vicuña Mackenna 4860, 782-0436 Macul, Santiago, Chile

20 Pontificia Universidad Católica de Chile, Av. Vicuña Mackenna 4860, 782-0436 Macul, Santiago, Chile

21 Instituto de Astrofísica e Ciências do Espaço, Universidade do Porto, CAUP, Rua das Estrelas, 4150-762 Porto, Portugal

Received 20 January 2017 / Accepted 16 February 2017

\begin{abstract}
We use Gaia-ESO (GES) Survey iDR4 data to explore the Mg-Al anti-correlation in globular clusters that were observed as calibrators, as a demonstration of the quality of Gaia-ESO Survey data and analysis. The results compare well with the available literature, within 0.1 dex or less, after a small (compared to the internal spreads) offset between the UVES and GIRAFFE data of $0.10-0.15$ dex was taken into account. In particular, for the first time we present data for NGC 5927, which is one of the most metal-rich globular clusters studied in the literature so far with $[\mathrm{Fe} / \mathrm{H}]=-0.39 \pm 0.04 \mathrm{dex}$; this cluster was included to connect with the open cluster regime in the Gaia-ESO Survey internal calibration. The extent and shape of the Mg-Al anti-correlation provide strong constraints on the multiple population phenomenon in globular clusters. In particular, we studied the dependency of the $\mathrm{Mg}$ - $\mathrm{Al}$ anti-correlation extension with metallicity, present-day mass, and age of the clusters, using GES data in combination with a large set of homogenized literature measurements. We find a dependency with both metallicity and mass, which is evident when fitting for the two parameters simultaneously, but we do not find significant dependency with age. We confirm that the Mg-Al anti-correlation is not seen in all clusters, but disappears for the less massive or most metal-rich clusters. We also use our data set to see whether a normal anticorrelation would explain the low $[\mathrm{Mg} / \alpha]$ observed in some extragalactic globular clusters, but find that none of the clusters in our sample can reproduce it; a more extreme chemical composition, such as that of NGC 2419, would be required. We conclude that GES iDR4 data already meet the requirements set by the main survey goals and can be used to study globular clusters in detail, even if the analysis procedures were not specifically designed for them.
\end{abstract}

Key words. surveys - stars: abundances - globular clusters: general - globular clusters: individual: NGC 5927

\footnotetext{
$\star$ Based on data products from observations made with ESO Telescopes at the La Silla Paranal Observatory under programme ID 188.B-3002.

$\star \star$ Full Table 2 is only available at the CDS via anonymous ftp to cdsarc.u-strasbg.fr $(130.79 .128 .5)$ or via

http://cdsarc.u-strasbg.fr/viz-bin/qcat?J/A+A/601/A112
} 


\section{Introduction}

The phenomenon of multiple populations in globular clusters (GCs) has been intensively studied in the last 20-30 yr, but we still lack a clear explanation of their origin (Gratton et al. 2012). The abundance variations pattern pinpoints the CNO-cycle burning of hydrogen as the major source of the phenomenon because most of the elements that are observed to vary in GCs are used as catalysts in various $\mathrm{CNO}$ sub-cycles, where they are depleted, or accumulated, depending on the particular reaction rates. However, a hot debate is still ongoing about which types of polluters convey the processed material into the GC insterstellar gas reservoir and how this material is recycled to pollute a fraction of the GC stars (see D'Ercole et al. 2008; Decressin et al. 2007; Larsen et al. 2012b; Renzini et al. 2015; Bastian et al. 2015, for references).

The Mg-Al anti-correlation is of particular importance because unlike those of $\mathrm{C}-\mathrm{N}$ and $\mathrm{Na}-\mathrm{O}$, its extension varies significantly from one GC to the other, to the point of disappearing completely in some GCs. $\mathrm{Mg}$ and $\mathrm{Al}$ are involved in the hot $\mathrm{Mg}$-Al cycle, which requires high temperatures $\left(\sim 10^{8} \mathrm{~K}\right.$; Denissenkov et al. 2015; Renzini et al. 2015) and, therefore, its study can place very strong constraints on the type of star that is responsible for the peculiar chemistry observed in GCs. Another advantage of studying $\mathrm{Mg}$ and $\mathrm{Al}$ is that they suffer much less internal mixing compared to $\mathrm{C}$ and $\mathrm{N}$, or even $\mathrm{Na}$ and $\mathrm{O}$, and, therefore, the observed abundances do not depend on the evolutionary status of a star.

The Gaia-ESO survey (GES; Gilmore et al. 2012; Randich et al. 2013), which is being carried out at the ESO VLT with FLAMES (Pasquini et al. 2000), observed GCs as calibrators for the astrophysical parameters (AP) and abundance ratios (Pancino \& the Gaia-ESO Survey collaboration 2017, hereafter P16). Part of the observed GCs were included in the fourth internal data release (iDR4) that is based on data gathered from December 2011 to July 2014 and from which the next GES public release will be published through the ESO archive system ${ }^{1}$. The iDR4 data also include relevant archival data obtained with FLAMES in the GES set-ups. A particular advantage of the adopted observing set-ups is that they allow for an accurate measurement of the $\mathrm{Mg}$ and $\mathrm{Al}$ abundance ratios with both the UVES and GIRAFFE spectrographs. Thus, GES provides statistical samples that are comparable to those recently obtained by APOGEE (Mészáros et al. 2015) and the FLAMES GC survey (Carretta et al. 2009a,b, 2011, 2013a, 2014).

The paper is organized as follows: in Sect. 2 we describe the data treatment and sample selection; in Sect. 3 we present the results and explore their robustness; in Sect. 4 we describe and discuss the behaviour of the $\mathrm{Mg}-\mathrm{Al}$ abundance variations; and in Sect. 5 we summarize our findings and conclusions.

\section{Data sample and treatment}

The GES iDR4 data on GCs are all based on the UVES setup centred around $5800 \AA$ and on the two GIRAFFE set-ups HR 10 (5339-5619 $\AA)$ and HR 21 (8484-9001 $\AA$ ). The selection of calibration targets, which include GCs, was described in detail by P16. Briefly, 14 GCs were selected to adequately cover the relevant metallicity range, from $[\mathrm{Fe} / \mathrm{H}] \simeq-2.5$ to -0.3 dex, 11 of which were analyzed in iDR4. A few less studied GCs were included at the beginning of the survey, owing to

\footnotetext{
1 http://archive.eso.org/cms.html
}

pointing constraints (see P16 for more details) and, in particular, the sample includes NGC 5927, one of the most metal-rich GCs available. The selection of stars was focussed on red giants, except in NGC 5927, where mostly red clump stars were selected because of the high differential reddening and the need to maximize cluster members. Stars already having GIRAFFE archival observations in the ESO archive were prioritized to increase the wavelength coverage by including the GES set-ups. Stars already observed with UVES were not repeated. A few fibres were dedicated to reobserve some GIRAFFE targets with UVES, and vice-versa, to allow for cross-calibration.

All iDR4 data were reduced as described in detail by Sacco et al. (2014) for spectra taken with UVES (Dekker et al. 2000) at high resolution $(R=\lambda / \delta \lambda \simeq 47000)$ and by Jeffries et al. (2014) for spectra taken with GIRAFFE (Pasquini et al. 2000) at intermediate resolution $(R \simeq 16000$ 20000 ). Briefly, the UVES pipeline (Modigliani et al. 2004) was used to process UVES spectra, performing the basic reduction steps. Additional data analysis was performed for UVES with specific software developed at the Arcetri Astrophysical Observatory. The GIRAFFE spectra were processed with a dedicated software developed at $\mathrm{CASU}^{2}$ (Cambridge Astronomy Survey Unit).

\subsection{Abundance analysis}

The GES abundance analysis of UVES spectra was described in detail by Smiljanic et al. (2014) and Casey et al. (in prep.), while the analysis of GIRAFFE spectra was described by Recio-Blanco et al. (in prep.). Both are carried out by many research groups, using several state-of-the-art techniques. Because of the GES complexity, the data analysis is performed iteratively in each internal data release (iDR), gradually adding not only new data in each cycle, but also new processing steps to take into account lessons learned in the previous iDRs (offsets or trends identified through early science projects) or to increase the number of elements measured (from molecules, or faint features), or finally by adding detail to the measurements (corrections for non-LTE, rotational velocities, veiling, and many more). This methodology allows for a better quantification of the internal and external systematics, which are evaluated in a process of homogeneization of all node results, producing the final GES recommended APs and abundance ratios, as described by P16 and Hourihane et al. (in prep.).

To make the GES data analysis as uniform as possible, the analysis of $\mathrm{F}, \mathrm{G}$, and $\mathrm{K}$ type stars relies on a common set of atmospheric models (the MARCS grid; Gustafsson et al. 2008), a common linelist (Heiter et al. 2015b), and - for those methods that require it - a common library of synthetic spectra (computed with MARCS models and based on the grid by de Laverny et al. 2012). The solar reference abundances adopted in this paper were those by Grevesse et al. (2007). As mentioned, iDR4 abundances are computed in the LTE regime, and only future releases will include non-LTE corrections. Moreover, the GES homogenous analysis relies on a rich set of calibrating objects (including GCs), selected as described by P16. In particular, the external calibration of FGK stars in iDR4 relies mostly on the Gaia benchmark stars (Jofré et al. 2014; Blanco-Cuaresma et al. 2014; Heiter et al. 2015a; Hawkins et al. 2016).

When comparing the iDR4 abundances obtained from UVES and GIRAFFE, small (i.e. comparable to the internal spreads) offsets in the abundance ratios were found $(\sim 0.10-0.15 \mathrm{dex}$,

\footnotetext{
2 http://www.ast.cam.ac.uk/ mike/casu/
} 


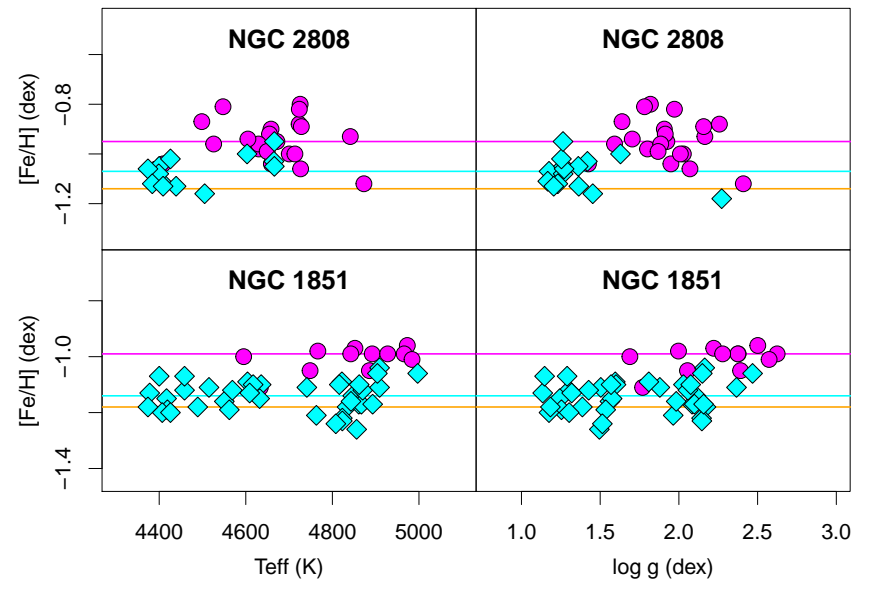

Fig. 1. Example of the small (compared to the errors and internal spreads) residual offsets in $[\mathrm{Fe} / \mathrm{H}]$ between UVES and GIRAFFE in GES iDR4 data, in two of the sample GCs: NGC 2808 (top panels) and NGC 1851 (bottom panels). The left panels show $[\mathrm{Fe} / \mathrm{H}]$ as a function of $T_{\text {eff }}$ and the right panels show $[\mathrm{Fe} / \mathrm{H}]$ as a function of $\log g$. The UVES stars are plotted as cyan symbols with their median $[\mathrm{Fe} / \mathrm{H}]$ as a cyan line. The GIRAFFE stars are plotted as magenta symbols with their median $[\mathrm{Fe} / \mathrm{H}]$ as a magenta line. The reference $[\mathrm{Fe} / \mathrm{H}]$ from Harris $(1996$, 2010 ) is plotted as an orange line.

depending on the GC), as shown by P16. For the present analysis, we reported the $[\mathrm{Fe} / \mathrm{H}]$ GIRAFFE measurements to the UVES scale using the difference between the median abundance of the two samples in each GC. We observed that once the $[\mathrm{Fe} / \mathrm{H}]$ offsets were corrected this way, there were no significant residual offsets when comparing the UVES and GIRAFFE measurements of the other elements considered in this paper. In any case, in the GES cyclic processing the recommended values of RVs, APs, and chemical abundances generally improve from one iDR to the next (see Randich et al., in prep., and P16). We thus expect the offsets to reduce considerably in future GES releases. Most importantly, as Fig. 1 shows, in iDR4 there are no significant trends of $[\mathrm{Fe} / \mathrm{H}]$ as a function of $T_{\text {eff }}$ or $\log g$ for either UVES or GIRAFFE results.

\subsection{Sample selection}

We applied the same quality selection criteria of the GES public release, which will be described in the ESO release documentation, to the iDR4 recommended results. For the cool giants in GCs, these criteria are $\delta T_{\text {eff }} / T_{\text {eff }}<5 \%, \delta \log g<0.3$ dex, and $\delta[\mathrm{Fe} / \mathrm{H}]<0.2$ dex. We also left out all stars that lacked AP or $\mathrm{RV}$ determinations.

We then selected GC probable members using the median $[\mathrm{Fe} / \mathrm{H}]$ and RV (following Lardo et al. 2015) as a reference for each GC, and removing all stars that deviated more than $3 \sigma$ from it. As discussed by P16, the GES median $[\mathrm{Fe} / \mathrm{H}]$ and RV generally agree with reference literature values (Harris 1996, 2010). The members selection was straightforward because the vast majority of field stars have roughly solar metallicity and RV approximately $0 \pm 50 \mathrm{~km} \mathrm{~s}^{-1}$, thus the GC stars differ significantly from field stars in at least one of $[\mathrm{Fe} / \mathrm{H}]$ or $\mathrm{RV}$.

The above selections lead to highly varying sample sizes for UVES and GIRAFFE, depending on several factors, such as spectral quality ( $\mathrm{S} / \mathrm{N}$ and spectral defects), observing conditions (sky and seeing), availability of previous information (photometry, membership, and other archival data), and cluster (crowding, GC compactness, distance, and metallicity). Of the 11 GCs included in iDR4 (see P16, for the selection criteria of calibrating objects) only 10 contained at least 5 red giants after the quality and membership selections. Of these, we excluded M 15 because the iDR4 analysis of its very metal-poor spectra did not provide satisfactory results. The final list of 9 analysed GCs is presented in Table 1, along with some relevant properties. The final sample contained 510 stars (159 with UVES and 351 with GIRAFFE) in $9 \mathrm{GCs}$ that had $\mathrm{Mg}$ or $\mathrm{Al}$ measurements. The stars and their relevant properties are listed in Table 2.

We stress again that the size and quality of the presented GC sample are comparable to the two largest GC surveys presented in the literature so far, i.e. the FLAMES GC survey and the APOGEE sample.

\section{Results}

\subsection{A quality control test on the $\mathrm{Na}-\mathrm{O}$ anti-correlation}

We started by comparing our results for the well-studied $\mathrm{Na}-\mathrm{O}$ anti-correlation with the FLAMES GC survey by Carretta et al. (2009a,b, 2011, 2013a, 2014), the 47 Tuc data by Cordero et al. (2014), the NGC 6752 study by Yong et al. (2005), and the M 2 studies by Yong et al. (2014) and Mészáros et al. (2015). We restricted the comparisons to high-resolution studies $(R>15000)$ of red giants. The results are plotted in Fig. 2, where only UVES measurements appear because oxygen is not included in the GES GIRAFFE set-ups.

As can be seen, the GES measurements agree well with the measurements in the literature in spite of the different methods, linelist selections, models, and data sets involved. The median offsets, measured by taking the difference between the median abundances obtained by GES and in the literature for each $\mathrm{GC}^{3}$, were in general lower than $\simeq 0.1 \mathrm{dex}$. For 47 Tuc, the GES data show less scatter in $[\mathrm{O} / \mathrm{Fe}]$ than in the literature, but they do not sample the full extension of $[\mathrm{Na} / \mathrm{Fe}]$; this is partly because of the quality selection criteria described in Sect. 2.2, which penalize oxygen abundances derived mostly from the weak [O I] line at $6300 \AA$. Also, the GES data for NGC 2808 show two wellseparated clumps of stars, while the literature data apparently display a more continuous distribution. We ascribe this to our small sample. Because this sample was randomly chosen, it included preferentially stars near the two most populated peaks of the underlying distribution, which contains five separate groups (Carretta 2015). The apparently continuous distribution of literature data is mostly driven by the GIRAFFE measurements (brown dots), which are more numerous but less precise than the UVES data (gold dots).

We present here for the first time $[\mathrm{Na} / \mathrm{Fe}]$ and $[\mathrm{O} / \mathrm{Fe}]$ abundance ratios for NGC 5927, one of the most-metal rich GC studied with high-resolution spectroscopy in the literature so far. NGC 5927 displays the same stubby Na-O anti-correlation as 47 Tuc, the other metal-rich GC in the sample. While the upper $[\mathrm{Na} / \mathrm{Fe}]$ limit is the same as any other GCs and is governed by the equilibrium abundance of the $\mathrm{NeNa}$ hot cycle, the lowest $[\mathrm{Na} / \mathrm{Fe}]$ abundances are slightly super-solar rather than subsolar, as expected for field stars at the same metallicity, as further discussed in Sect. 4.1.

In conclusion, the presented comparison confirms that the atmospheric parameters resulting from the GES homogenized analysis are well determined (see also P16).

\footnotetext{
3 In many cases, the stars in common between GES and the literature are too few or missing, therefore we preferred to use the differences between the median of each sample.
} 
Table 1. Basic properties of the GC sample.

\begin{tabular}{lcccccc}
\hline \hline Cluster & $\begin{array}{c}{[\mathrm{Fe} / \mathrm{H}]_{\mathrm{H} 96}} \\
(\mathrm{dex})\end{array}$ & $\begin{array}{c}\mathrm{RV}_{\mathrm{H} 96} \\
\left(\mathrm{~km} \mathrm{~s}^{-1}\right)\end{array}$ & $\begin{array}{c}\log \left(M / M_{\odot}\right) \\
(\mathrm{dex})\end{array}$ & $\begin{array}{c}{[\mathrm{Fe} / \mathrm{H}]_{\mathrm{GES}}} \\
(\mathrm{dex})\end{array}$ & $\begin{array}{c}\mathrm{RV}_{\mathrm{GES}} \\
\left(\mathrm{km} \mathrm{s}^{-1}\right)\end{array}$ & $N_{\star}$ \\
\hline NGC 104 (47 Tuc) & -0.72 & -18.0 & $6.05 \pm 0.04^{a}$ & $-0.71 \pm 0.02$ & $-17.6 \pm 0.8$ & 119 \\
NGC 362 & -1.36 & 223.5 & $5.53 \pm 0.04^{a}$ & $-1.12 \pm 0.03$ & $222.3 \pm 0.6$ & 73 \\
NGC 1851 & -1.18 & 320.5 & $5.49 \pm 0.04^{a}$ & $-1.07 \pm 0.04$ & $320.2 \pm 0.5$ & 89 \\
NGC 1904 (M 79) & -1.60 & 205.8 & $5.20 \pm 0.04^{a}$ & $-1.51 \pm 0.03$ & $205.2 \pm 0.5$ & 30 \\
NGC 2808 & -1.14 & 101.6 & $5.93 \pm 0.05^{a}$ & $-1.03 \pm 0.03$ & $103.7 \pm 1.4$ & 45 \\
NGC 4833 & -1.85 & 200.2 & $5.20 \pm 0.21^{b}$ & $-1.92 \pm 0.03$ & $200.6 \pm 1.0$ & 28 \\
NGC 5927 & -0.49 & -107.5 & $5.32 \pm 0.21^{b}$ & $-0.39 \pm 0.04$ & $-102.5 \pm 0.7$ & 85 \\
NGC 6752 & -1.54 & -26.7 & $5.16 \pm 0.21^{b}$ & $-1.48 \pm 0.04$ & $-26.3 \pm 0.7$ & 57 \\
NGC 7089 (M 2) & -1.65 & -5.3 & $5.84 \pm 0.05^{a}$ & $-1.47 \pm 0.03$ & $-1.8 \pm 1.3$ & 46 \\
\hline
\end{tabular}

Notes. The table lists: $[\mathrm{Fe} / \mathrm{H}]$ and RV from Harris (1996, 2010); the present-day mass from McLaughlin \& van der Marel (2005, annotated with ${ }^{a}$ ) or from Mandushev et al. (1991, annotated with ${ }^{b}$ ); the median $[\mathrm{Fe} / \mathrm{H}]$ and RV from GES data; and the number of GES member stars analyzed.

Table 2. List of the 510 stars that were selected from GES iDR4 as probable members and analyzed in this paper.

\begin{tabular}{lccccccccccc}
\hline \hline CNAME & Cluster & $\begin{array}{c}T_{\text {eff }} \\
(\mathrm{K})\end{array}$ & $\begin{array}{c}\delta T_{\text {eff }} \\
(\mathrm{K})\end{array}$ & $\begin{array}{c}\log g \\
(\mathrm{dex})\end{array}$ & $\begin{array}{c}\delta \log g \\
(\mathrm{dex})\end{array}$ & $\begin{array}{c}{[\mathrm{Fe} / \mathrm{H}]} \\
(\mathrm{dex})\end{array}$ & $\begin{array}{c}\delta[\mathrm{Fe} / \mathrm{H}] \\
(\mathrm{dex})\end{array}$ & $\begin{array}{c}\log \epsilon_{\mathrm{Al}} \\
(\mathrm{dex})\end{array}$ & $\begin{array}{c}\delta \log \epsilon_{\mathrm{Al}} \\
(\mathrm{dex})\end{array}$ & $\begin{array}{c}\log \epsilon_{\mathrm{Mg}} \\
(\mathrm{dex})\end{array}$ & $\begin{array}{c}\delta \log \epsilon_{\mathrm{Mg}} \\
(\mathrm{dex})\end{array}$ \\
\hline $12593863-7051321$ & NGC 4833 & 4673 & 124 & 1.308 & 0.246 & -1.844 & 0.103 & 5.61 & 0.07 & 5.94 & 0.13 \\
$13000316-7053486$ & NGC 4833 & 4675 & 132 & 1.207 & 0.239 & -1.920 & 0.106 & 5.60 & 0.07 & 5.59 & 0.13 \\
$12585746-7053278$ & NGC 4833 & 4678 & 127 & 1.316 & 0.261 & -2.024 & 0.119 & 5.29 & 0.07 & 5.73 & 0.14 \\
$12592040-7051156$ & NGC 4833 & 4623 & 123 & 1.130 & 0.252 & -1.922 & 0.101 & 5.55 & 0.07 & 5.50 & 0.13 \\
$12593089-7050304$ & NGC 4833 & 4613 & 123 & 1.112 & 0.254 & -1.920 & 0.108 & 5.55 & 0.07 & 5.66 & 0.14 \\
$12594306-7053528$ & NGC 4833 & 4635 & 117 & 1.070 & 0.235 & -1.890 & 0.111 & 5.61 & 0.07 & 5.69 & 0.13 \\
\hline
\end{tabular}

Notes. The table is available in its entirety at the CDS, here we show a portion to illustrate its contents. The reported errors are the result of the complex GES homogenization procedure and thus include random and systematic error sources.

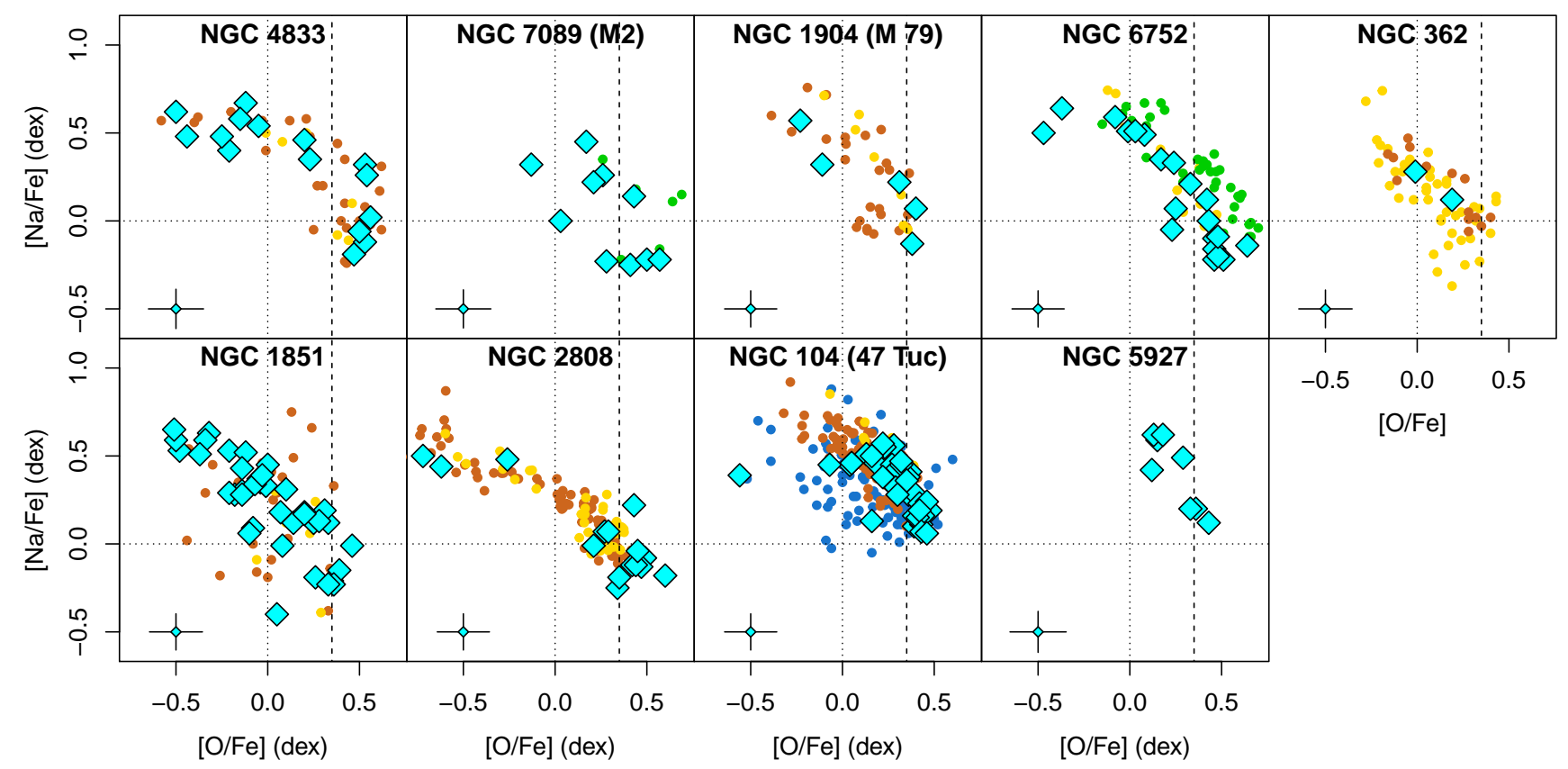

Fig. 2. Na-O anti-correlation. Panels show different GCs, sorted by increasing metallicity from left to right and from top to bottom. Dotted lines indicate the solar abundance ratios, dashed lines the typical halo $\alpha$-enhancement. The GES UVES data are plotted as cyan diamonds; literature data from the FLAMES GC survey are plotted in brown for GIRAFFE and in gold for UVES; NGC 6752 and M 2 data by Yong et al. (2005) and Yong et al. (2014) are plotted in green; the 47 Tuc analysis by Cordero et al. (2014) is plotted in blue. Typical (median) error bars are reported in the bottom left corner of each panel.

\subsection{Mg-Al anti-correlation}

The Mg-Al anti-correlation for the selected iDR4 stars is plotted in Fig. 3, along with the available literature data. In contrast to the Na-O anti-correlation, we present both UVES and GIRAFFE measurements. Our measurements compare well with the literature with small offsets that are $<0.1$ dex, i.e. within the quoted errors, as in the $\mathrm{Na}-\mathrm{O}$ case. 


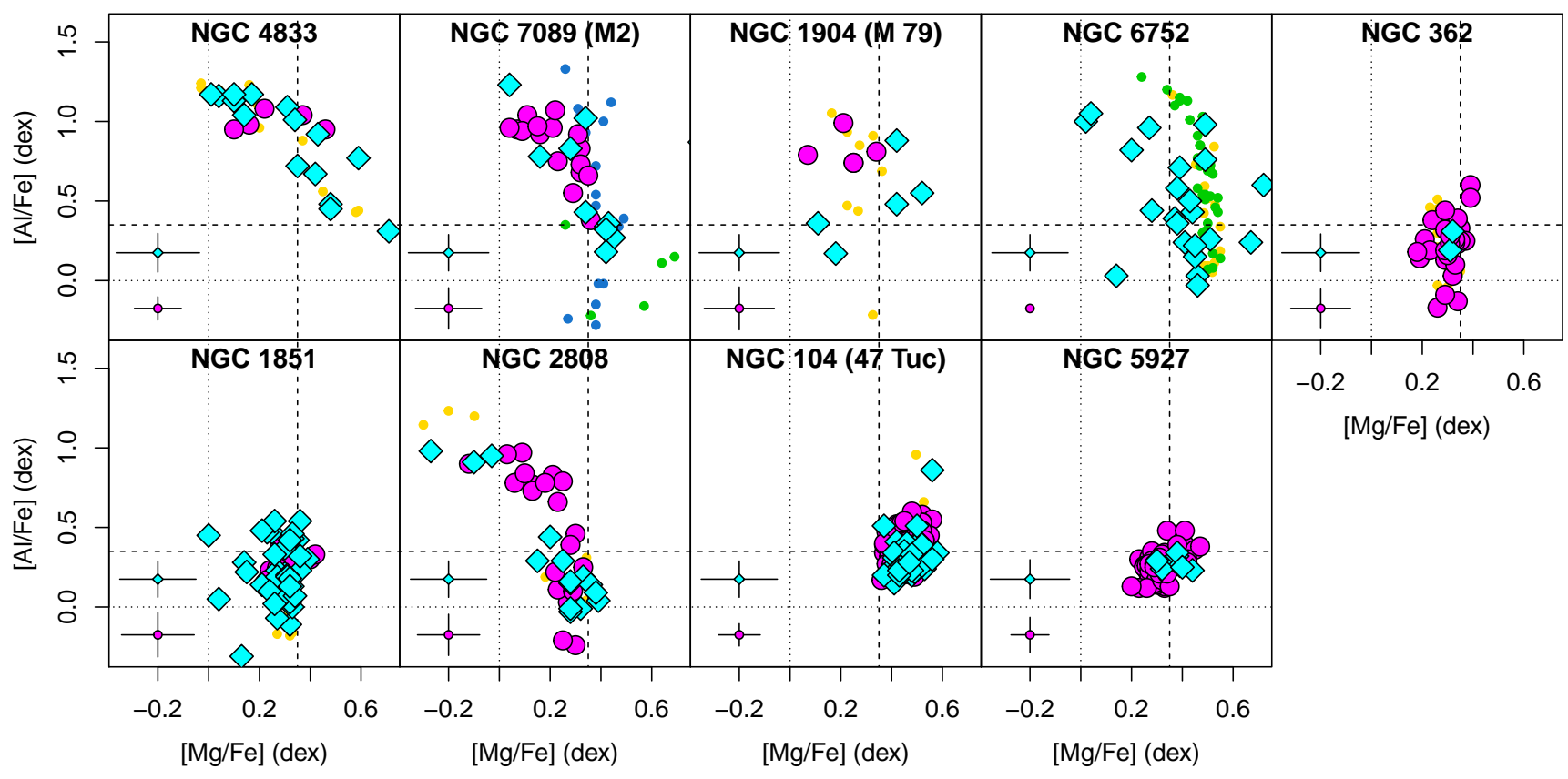

Fig. 3. Mg-Al anti-correlation. Panels show different GCs, sorted by increasing metallicity from left to right and from top to bottom. Dotted lines indicate the solar abundance ratios, dashed lines the typical halo $\alpha$-enhanced ratios. The GES UVES data are plotted as cyan diamonds; GIRAFFE data as magenta circles; UVES literature data from the FLAMES GC survey are plotted in gold; NGC 6752 and M 2 data by Yong et al. (2005 and 2014) are plotted in green; M 2 data by Mészáros et al. (2015) are plotted in blue. Typical (median) error bars are reported on the bottom left corner of each panel.

For NGC 1904 there are few stars and they appear very scattered. For the other $8 \mathrm{GCs}$, however, we clearly see that the $\mathrm{Mg}-\mathrm{Al}$ anti-correlation has a variable extension. Four GCs have a well-developed and curved $\mathrm{Mg}$-Al anti-correlation, i.e. NGC 2808, NGC 4833, NGC 6752, and M 2. Two GCs have a stubby Mg-Al distribution, i.e. NGC 362 and NGC 1851, which mostly display an $[\mathrm{Al} / \mathrm{Fe}]$ spread and no significant $[\mathrm{Mg} / \mathrm{Fe}]$ spread. The two most metal-rich GCs in the sample, 47 Tuc and NGC 5927, show no clear signs of an anti-correlation. This behaviour was already noted by Carretta et al. (2009a), who explicitly mentioned the GC present-day mass and metallicity as the two main parameters driving the extent of the $\mathrm{Mg}$ - $\mathrm{Al}$ anticorrelation (see Sect. 4 for more discussion on this point).

We did not detect any significant variation of the combined abundance of $\mathrm{Mg}$ and $\mathrm{Al}$. This is consistent with no net production of these elements, but just the result of the conversion of $\mathrm{Mg}$ into $\mathrm{Al}$ during the $\mathrm{Mg}-\mathrm{Al}$ cycle. Concerning the $\mathrm{Al}-\mathrm{Si}$ branch of the Mg-Al cycle (see also Yong et al. 2005; Carretta et al. 2009a), we looked for Si variations in our sample, but unfortunately GES iDR 4 contains only a few Si measurements that pass all the criteria employed to select the sample stars. Inspection of the $[\mathrm{Si} / \mathrm{Fe}]$ ratio as a function of $[\mathrm{Al} / \mathrm{Fe}]$ or $[\mathrm{Mg} / \mathrm{Fe}]$ for the few stars with reliable Si measurements in iDR4 did not reveal any clear trend.

\section{Discussion}

To put our results in context, we combined the GES iDR4 data with the FLAMES GC survey (Carretta et al. 2009a,b) and the APOGEE survey (Mészáros et al. 2015) measurements. Literature data were shifted in both $[\mathrm{Fe} / \mathrm{H}]$ and the $[\mathrm{El} / \mathrm{Fe}]$ abundance ratios by small amounts $(\leq 0.1 \mathrm{dex})$ to place them on the GES iDR4 scale. The shifts were computed using the median values of key elements for the GCs in common among studies ${ }^{4}$. The combined sample contains $\simeq 1300$ stars in 28 GCs with both $\mathrm{Mg}$ and $\mathrm{Al}$ measurements, or 2500 stars if one also counts the stars having $\mathrm{Na}$ or $\mathrm{O}$, but missing either the $\mathrm{Mg}$ or $\mathrm{Al}$ measurement.

In the next sections, we discuss some of the Mg-Al anticorrelation properties that were apparent during a preliminary exploration of the combined sample. We leave the discussion of other elements to the following GES releases, where more stars, more GCs, and more elements will be available, and the whole GES intercalibration procedure will be more refined.

\subsection{Comparison with field stars}

We started by examining the $\mathrm{Na}-\mathrm{O}$ and $\mathrm{Mg}-\mathrm{Al}$ anti-correlation as a function of metallicity, and we compared the available GC measurements with the Milky Way (MW) field population. Because iDR4 contains mostly MW stars with $[\mathrm{Fe} / \mathrm{H}] \geq$ -1.0 dex, we added metal-poor stars extracted from the SAGA database (Suda et al. 2008). Figure 4 shows the comparisons. Oxygen measurements in iDR4 are very spread out, because they are mostly based on the weak [O I] line at $6300 \AA$, contaminated by the telluric $\mathrm{O}_{2}$ band, and because they rely on the generally lower S/N of field star spectra compared to GC stars (see P16 for details), but the bulk measurements follow the expected trend. In spite of the heterogeneity of the sample and of our relatively simple homogeneization method, the agreement among the plotted studies is remarkably good.

4 We had M 2 in common with the APOGEE survey and six GCs in common with the FLAMES GC survey (see also Figs. 2 and 3). The handful of stars in common among the various studies were not sufficient to compute reliable shifts, and were removed from the sample, retaining with precedence the GES data, then the APOGEE, and then the FLAMES GC survey data. 


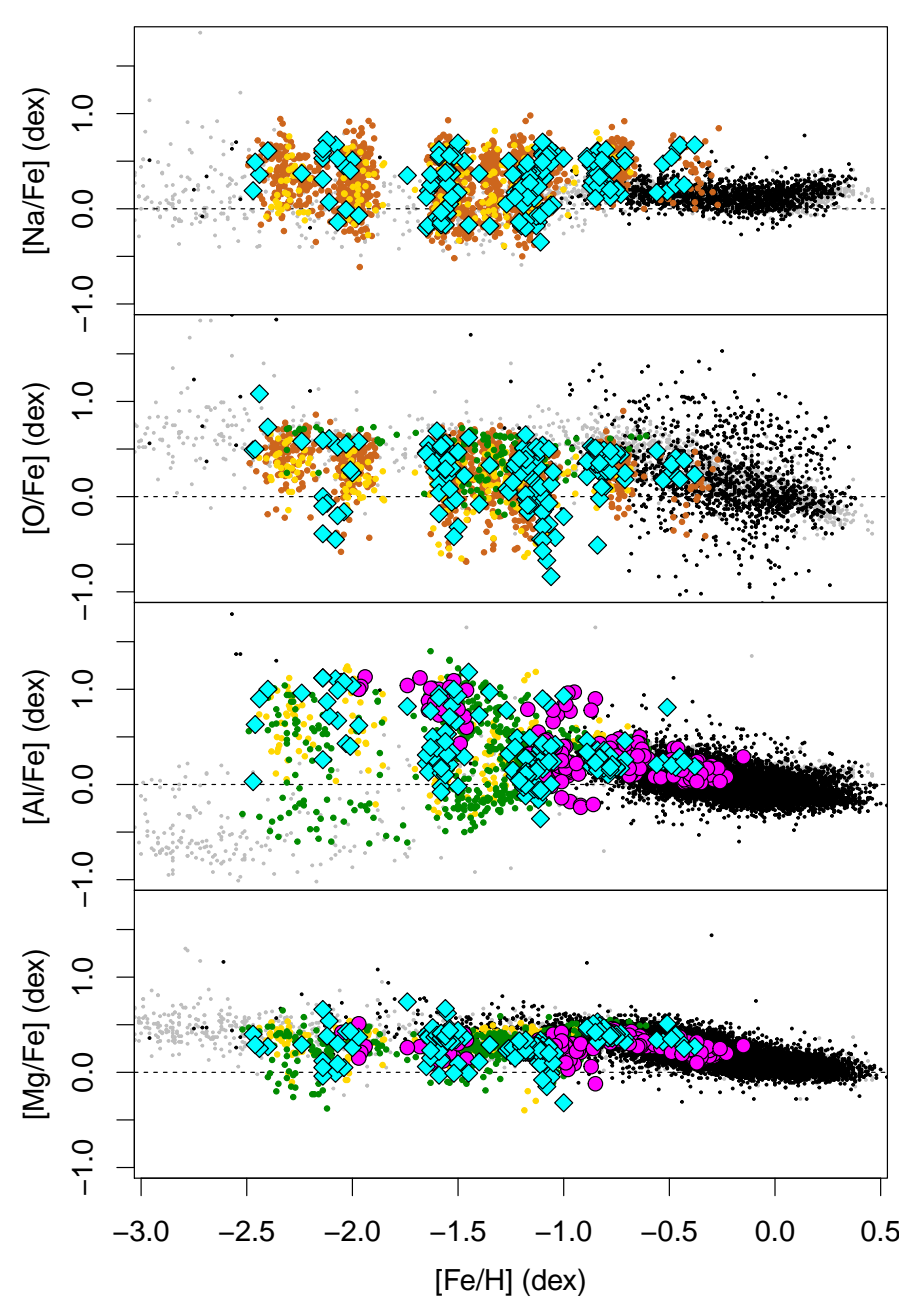

Fig. 4. Run of the four main anti-correlated elements as a function of $[\mathrm{Fe} / \mathrm{H}]$. For MW field stars, GES iDR4 results are plotted as black dots and SAGA metal-poor stars as grey dots. Homogenized APOGEE data are plotted in green and FLAMES GC surveys data are plotted in yellow for UVES and brown for GIRAFFE. GES iDR4 measurements are plotted in cyan for UVES and magenta for GIRAFFE.

Two important things should be noted at this point. The first is that both GES and the FLAMES GC survey use similar instrumental set-ups, wavelength ranges, and S/N. The GES targets mostly MW field stars of higher metallicity, while the FLAMES $\mathrm{GC}$ survey was focussed on the $\mathrm{Na}-\mathrm{O}$ anti-correlation. As a result, neither of these surveys contains many measurements at $[\mathrm{Fe} / \mathrm{H}] \leq-1.7 \mathrm{dex}$, and in particular, they do not contain many stars with low values of $[\mathrm{Al} / \mathrm{Fe}]$ or $[\mathrm{Mg} / \mathrm{Fe}]^{5}$ because they mostly rely on spectral lines that become weak at those metallicities. On the contrary, APOGEE measurements are obtained with a different wavelength range and using different features and selection criteria, and therefore that sample contains many more stars with low $\mathrm{Al}$ or $\mathrm{Mg}$, as can be seen in Fig. 4. On the other hand, GES data add NGC 5927 to the sample, extending the $[\mathrm{Fe} / \mathrm{H}]$ coverage to $[\mathrm{Fe} / \mathrm{H}]=-0.49$ dex, while the two previous systematic studies considered here reached $[\mathrm{Fe} / \mathrm{H}] \simeq-0.7$ dex with 47 Tuc and $\mathrm{M} 71$.

As was noted by others before, the lower boundary of the $\mathrm{Na}$ and $\mathrm{Al}$ distribution in GCs is aligned with the typical field star value at any given metallicity. Similarly, the upper boundary

\footnotetext{
5 Both GES and the FLAMES GC survey contain several upper limits in the most metal-poor GCs, which are not plotted in this paper.
}

of the $\mathrm{O}$ and $\mathrm{Mg}$ distribution in GCs is aligned with the typical field-star $\alpha$-enhancement at any given metallicity. This supports the idea that the main contributors to the chemistry of normal stars in GCs (often called first generation stars or unenriched stars) are mostly SNe II, such as for the field stars at the same metallicity with $\mathrm{SNe}$ Ia intervening only above $[\mathrm{Fe} / \mathrm{H}] \simeq-1.0 \mathrm{dex}$.

The abundance of anomalous stars (often called second generation or enriched stars) is thought to be governed by CNO cycle processing at high temperatures (Kraft 1994; Gratton et al. 2004). The extent of $\mathrm{Na}$ variations in GC stars changes slightly with $[\mathrm{Fe} / \mathrm{H}]$. This is mostly governed by the lower boundary variations of $[\mathrm{Na} / \mathrm{Fe}]$ in $\mathrm{GC}$ stars, which follow the field population behaviour as discussed. The upper boundary - governed by the equilibrium abundances reached in the Ne-Na cycle - shows only moderate variations in our sample, which are roughly at $[\mathrm{Na} / \mathrm{Fe}] \simeq+0.6 \mathrm{dex}$ and contained within $\pm 0.15 \mathrm{dex}^{6}$. The extent of $[\mathrm{Al} / \mathrm{Fe}]$ variations in $\mathrm{GC}$ stars, instead, changes dramatically with $[\mathrm{Fe} / \mathrm{H}]$ both in the upper and lower boundaries. While it was suggested that $[\mathrm{Fe} / \mathrm{H}]$ is not the sole parameter governing $\mathrm{Al}$ variations (see also Sect. 4.2), the Al spread clearly varies with metallicity, from a maximum of $\Delta[\mathrm{Al} / \mathrm{Fe}] \simeq 1.5 \mathrm{dex}$ and more below $[\mathrm{Fe} / \mathrm{H}] \simeq-1.0 \mathrm{dex}$, to $\Delta[\mathrm{Al} / \mathrm{Fe}] \leq 0.5 \mathrm{dex}$ above that metallicity, where the spread becomes compatible with measurement uncertainties.

These considerations lead us to believe that the entire sample of 1300 stars should be used when studying the behaviour of the $\mathrm{Mg}-\mathrm{Al}$ anti-correlation with GC properties to increase the parameter coverage and the statistical significance of the analysis. Figure 4 is an example of the striking power of such a sample, and reveals the importance of $[\mathrm{Fe} / \mathrm{H}]$ as a driving parameter for the presence and extent of the $\mathrm{Mg}-\mathrm{Al}$ anti-correlation.

\section{2. $M g-A l$ anti-correlation extension}

We have seen that a clear variation of the $[\mathrm{Al} / \mathrm{Fe}]$ spread with $[\mathrm{Fe} / \mathrm{H}]$ is apparent in Fig. 4, and this is not only caused by the natural $[\mathrm{Al} / \mathrm{Fe}]$ variations observed for field stars (the lower $[\mathrm{Al} / \mathrm{Fe}]$ boundary). The question of which GC properties govern the extension (or presence) of the $\mathrm{Mg}$ - $\mathrm{Al}$ anti-correlation has been explored previously in the literature (see e.g. Carretta et al. 2009a,b; Mészáros et al. 2015; Cabrera-Ziri et al. 2016, for example). Both $[\mathrm{Fe} / \mathrm{H}]$ and present-day mass were mentioned as the most important parameters in those works. However, when only $[\mathrm{Fe} / \mathrm{H}]$ was considered (Fig. 4 by Cabrera-Ziri et al. 2016), only weak correlations were found, with large spreads and unclear statistical significance. In that case, 25 GCs were examined with typically 10-20 stars per GC. Here, we can profit from our combined sample of 28 GCs with $\simeq 50$ stars each on average, as described in Sect. 4.1, and re-examine these parameters as drivers of the $\mathrm{Mg}-\mathrm{Al}$ anti-correlation.

We therefore proceeded to fit the data using two different indicators of the anti-correlation extension: the standard deviation of the $[\mathrm{Al} / \mathrm{Mg}]$ distribution and its maximum variation, i.e. the difference between the maximum and minimum values of $[\mathrm{Al} / \mathrm{Mg}]$ for each $\mathrm{GC}$. The two indicators are expressed as $\sigma[\mathrm{Al} / \mathrm{Mg}]$ and $\Delta[\mathrm{Al} / \mathrm{Mg}]$ in the following ${ }^{7}$. Figure 5 shows the

6 An extremely homogeneous and populous sample would be required to better quantify this important aspect.

7 Both indicators are subject to measurement and statistical biases. Measurement effects (most notably outliers) tend to produce an overstimate of the Mg-Al extension, while sampling effects (small sample sizes) tend to produce an underestimate. 


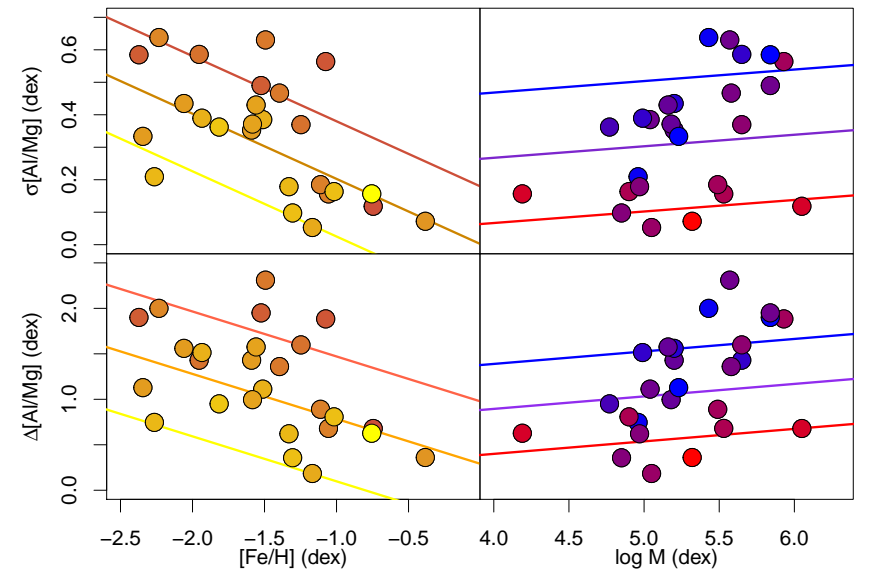

Fig. 5. Extent of the $\mathrm{Mg}-\mathrm{Al}$ anti-correlation, measured as $\sigma[\mathrm{Al} / \mathrm{Mg}]$ (top panels) and $\Delta[\mathrm{Al} / \mathrm{Mg}]$ (bottom panels), based on the sample described in the text. The behaviour as a function of average $[\mathrm{Fe} / \mathrm{H}]$ (left panels) and total $\log M$ (present-day mass, right panels) of each GC is shown. The GCs in the left panels are coloured as a function of $\log M$, where yellow corresponds to $\log M=4.19$ dex (the lowest mass in the sample) and dark orange to $\log M=6.05$ dex (the highest mass). In the right panels, points are coloured as a function of their metallicity with red corresponding to $[\mathrm{Fe} / \mathrm{H}]=-0.5 \mathrm{dex}$ (the highest metallicity in the sample) and blue to $[\mathrm{Fe} / \mathrm{H}]=-2.5$ dex (the lowest metallicity). Our models in the form $a[\mathrm{Fe} / \mathrm{H}]+b \log M+c$ are also plotted as lines coloured based on mass or metallicity.

results graphically, where it is apparent that the most massive GCs tend to have higher values with both indicators in the plot as a function of $[\mathrm{Fe} / \mathrm{H}]$, and the most metal-poor GCs also have higher spread in the plot as a function of $\log M$. If we were to fit the two parameters separately, we would obtain very high spreads and very weak relations even with our larger sample.

We therefore employed a linear fit on both parameters simultaneously and we obtained the following results:

$$
\begin{aligned}
\sigma[\mathrm{Al} / \mathrm{Mg}]= & 0.19( \pm 0.06) \log M-0.20( \pm 0.05)[\mathrm{Fe} / \mathrm{H}] \\
& -0.94( \pm 0.33) \\
\Delta[\mathrm{Al} / \mathrm{Mg}]= & 0.67( \pm 0.21) \log M-0.53( \pm 0.17)[\mathrm{Fe} / \mathrm{H}] \\
& -3.16( \pm 1.11) .
\end{aligned}
$$

The fits are also reported in Fig. 5. The $p$ values of the $\sigma[\mathrm{Al} / \mathrm{Mg}]$ and $\Delta[\mathrm{Al} / \mathrm{Mg}]$ are 0.0001493 and 0.0005242 , respectively, suggesting that it would be improbable to obtain the observed distribution by chance (if the chosen model $^{8}$ was correct). The errors on the coefficient are also relatively low, suggesting that the twoparameter linear model is a reasonable description of the data. We thus can conclude that both parameters ${ }^{9}$ are indeed important in determining the extension of the $\mathrm{Mg}$ - $\mathrm{Al}$ anti-correlation in the sense that we find much smaller extensions for GCs that are metal rich or less massive (or both). This also supports the results obtained by Carretta et al. (2010) on the Na-O data of the FLAMES GC survey, and the photometric analysis carried out by Milone et al. (2017).

This does not mean that the model we adopted is the best one, and it does not mean that $[\mathrm{Fe} / \mathrm{H}]$ and $\log M$ are the only two parameters at play, especially considering that the errors on the

\footnotetext{
8 Here and in the following, we use the word model in the statistical sense, i.e. a way of describing the data phenomenologically and not a physical model.

9 It is important to stress at this point that no mass-metallicity relation is apparent in Galactic GCs.
}

derived coefficients are of about $\simeq 30 \%$; also the residual distributions, although centered on zero, have relatively large spreads of $\operatorname{med}\left(\mathrm{rms}_{\Delta[\mathrm{Al} / \mathrm{Mg}]}\right)=-0.005 \pm 0.768$ and $\operatorname{med}\left(\mathrm{rms}_{\sigma[\mathrm{Al} / \mathrm{Mg}]}\right)=$ $+0.014 \pm 0.258$. In the present analysis, we have not used the errors in the fit because of the heterogenity of the data sources and therefore of error determinations, but even accounting for that, the relatively large spreads could point towards some extra parameter. We also tried a different model, adding a quadratic term in both $[\mathrm{Fe} / \mathrm{H}]$ and $\log M$, but the fit did not improve significantly. Similarly, when adding the age parameter from Marín-Franch et al. (2009) or from VandenBerg et al. (2013) as a third linear term, the coefficient was always low $(<0.0001)$, and the quality of the fit was worse than that of the two-parameter fit. A full statistical analysis of the relation between anti-correlation parameters and GC properties will be presented in a forthcoming paper when the analysis of the whole GES sample of stars in all the observed GCs has been completed; we will also have data on the $[\mathrm{C} / \mathrm{Fe}]$ and $[\mathrm{N} / \mathrm{Fe}]$ ratios.

The $\mathrm{Mg}-\mathrm{Al}$ anti-correlation is a problem for the scenarios based on fast rotating massive stars (FMRS; Decressin et al. 2007) or massive interacting binaries (MIB; de Mink et al. 2009), which activate CNO burning in their cores but require very high masses (well above $100 M_{\odot}$ ) and some tweaking of the reaction rates to reproduce the $\mathrm{Mg}-\mathrm{Al}$ observations. More massive stars would be required, such as super-massive stars (SMS; $\sim 1000 M_{\odot}$, Denissenkov et al. 2015), but these are not observed and therefore their postulated physics is highly uncertain. We expect a metallicity dependency for SMS because of the strong wind mass loss (Vink et al. 2011) that would lead to the formation of smaller SMS at higher metallicity. Asymptotic giant branch polluters (AGB), which activate CNO burning in the shell and also hot-bottom burning at high masses, can naturally explain the $\mathrm{Mg}-\mathrm{Al}$ observations because both the depletion of $\mathrm{Mg}$ and the production of $\mathrm{Al}$ are extremely sensitive to the AGB star metallicity (Ventura et al. 2016). However, none of the scenarios presented in the literature so far is entirely free from serious shortcomings (Renzini et al. 2015). We also remark that no conclusive answer can be drawn by considering one anticorrelation only and this, like other works, has to be considered as a preliminary exploration.

The correlation of the Mg-Al extent with present-day GC mass has not been explained in detail in any of the scenarios proposed so far. It would be necessary to explore whether the observed mass variations among Galactic GCs (presently in the range $10^{4}-10^{6} M_{\odot}$ ) are sufficient to significantly change the ability of the forming GCs (with their unknown initial masses) to retain the polluters ejecta.

\subsection{Low-Mg in extragalactic GCs}

It was reported by various authors (Larsen et al. 2014; Colucci et al. 2014; Sakari et al. 2015) that the integrated light, high-resolution abundance determinations of extragalactic GCs tend to have $[\mathrm{Mg} / \mathrm{Fe}]$ significantly below that of MW GCs, around $[\mathrm{Mg} / \mathrm{Fe}] \simeq 0 \mathrm{dex}$ and lower, rather than $0.3-0.4 \mathrm{dex}$. This observational fact is difficult to explain with problems in the abundance analysis alone; the comparison by Colucci et al. (2017) highlights an underestimate of $[\mathrm{Mg} / \mathrm{Fe}]$ of $\simeq 0.2 \mathrm{dex}$ with integrated light spectroscopy for some Galactic GC, while Larsen et al. (2017) find systematic effects of 0.1 dex at most. The $\mathrm{Mg}$ underabundance is not seen in other $\alpha$-element abundances that are consistent with the typical $\alpha$-enhancement expected from metal-poor GCs in the respective galaxies. In other 
words, $[\mathrm{Mg} / \alpha]$ in these metal-poor, extragalactic GCs is lower than in MW GCs with similar metallicity.

Figures 3 and 4 show that some Galactic GCs - not all contain a fraction of stars well below $[\mathrm{Mg} / \mathrm{Fe}] \simeq 0 \mathrm{dex}$. The question then is whether the fraction of low-Mg stars and the $\mathrm{Mg}$ spread caused by a normal $\mathrm{Mg}-\mathrm{Al}$ anti-correlation would be sufficient to produce an average $\mathrm{GC}\langle[\mathrm{Mg} / \mathrm{Fe}]\rangle$ close to solar or even lower, as observed in extragalactic GCs (Larsen 2016). While a deeper investigation of this topic is outside the scope of the present paper, we can use the collected GES and literature samples to understand if anti-correlations are at least a viable explanation for the observed low $[\mathrm{Mg} / \alpha]$ abundances in many extragalactic GCs. In practice, we averaged the $[\mathrm{Mg} / \alpha]$ measurements for stars in each GC, which is appropriate because they are based on relatively weak absorption lines, but can be an incomplete representation of the abundance in the whole GC and on the proportions of stars with different $\mathrm{Mg}$ content. Integrated light measurements, on the other hand, represent a complete average - weighted by star brightness and cut by limiting magnitude - of a GC (see Colucci et al. 2017; Larsen et al. 2017, for a comparison between the two methods).

We collected literature data on extragalactic GCs in M 31 (Colucci et al. 2009, 2014; Sakari et al. 2015), the LMC (Large Magellanic Cloud; Mucciarelli et al. 2008, 2009, 2010, 2014; Johnson et al. 2006; Mateluna et al. 2012), the Fornax dwarf galaxy (Letarte et al. 2006; Larsen et al. 2012a), and WLM (Wolf-Lundmark-Melotte galaxy; Larsen et al. 2014). To illustrate the effect, we plotted the data for extragalactic GCs together with the MW field samples and the Galactic GCs from the collection described in the previous section (Fig. 6). The figure shows the average or integrated abundance of each GC, where the $\alpha$-elements are represented by $\mathrm{Ca}$ and $\mathrm{Si}$, which are present in all the used studies. As can be noticed, many extragalactic GCs have normal $\alpha$-enhancement but low $[\mathrm{Mg} / \mathrm{Fe}]$, and as a result their $[\mathrm{Mg} / \alpha]$ ratios are below zero. The MW GCs, however, all have $[\mathrm{Mg} / \mathrm{Fe}] \simeq 0.4 \mathrm{dex}-$ with very few exceptions - and have a spread that is compatible with the errors and the internal Mg spread of Fig. 4.

To our knowledge, the only Galactic GC that contains a sufficient percentage of stars $(\simeq 50 \%)$ with a sufficiently low $[\mathrm{Mg} / \mathrm{Fe}]$ is NGC 2419 (Mucciarelli et al. 2012), reaching as low as $[\mathrm{Mg} / \mathrm{Fe}] \simeq-1.0$ dex. Based on the complicated chemistry of NGC 2419, it was suggested that it has extragalactic origin (Mucciarelli et al. 2012; Cohen \& Kirby 2012; Carretta et al. 2013b; Ventura et al. 2012), which would fit the observed data trend. On the other hand, Rup 106, which is known to have low $[\mathrm{Mg} / \mathrm{Fe}]$ (Villanova et al. 2013), has a perfectly normal $[\mathrm{Mg} / \alpha]$ because its stars are not $\alpha$-enhanced. We conclude that it is difficult to explain the low integrated $[\mathrm{Mg} / \alpha]$ values of many extragalactic GCs with the typical $\mathrm{Mg}-\mathrm{Al}$ anti-correlation observed in Galactic GCs. A more extreme $\mathrm{Mg}$ depletion and a larger percentage of stars with such low $\mathrm{Mg}$ would be required, similar to what observed in NGC 2419.

Apart from the extreme morphology of the $\mathrm{Mg}-\mathrm{Al}$ anticorrelation observed, for example in NGC 2419 (an internal effect), there is an additional explanation for the low average $[\mathrm{Mg} / \alpha]$ of some extragalctic GCs that is linked to the global chemical evolution of their host galaxies (an external effect). It has been observed that in dwarf galaxies $[\mathrm{Mg} / \mathrm{Fe}]$ is lower than the average $\alpha$-enhancement for stars close to the "knee" of the $[\alpha / \mathrm{Fe}]$ trend. This was explained considering that $\mathrm{SNe} \mathrm{Ia}$ produce some amounts of $\mathrm{Ca}, \mathrm{Si}$, and $\mathrm{Ti}$ but not $\mathrm{Mg}$, which is produced only by SNe II (Tsujimoto \& Bekki 2012). In that case, we should observe a progressively lower $[\mathrm{Mg} / \alpha]$ in the

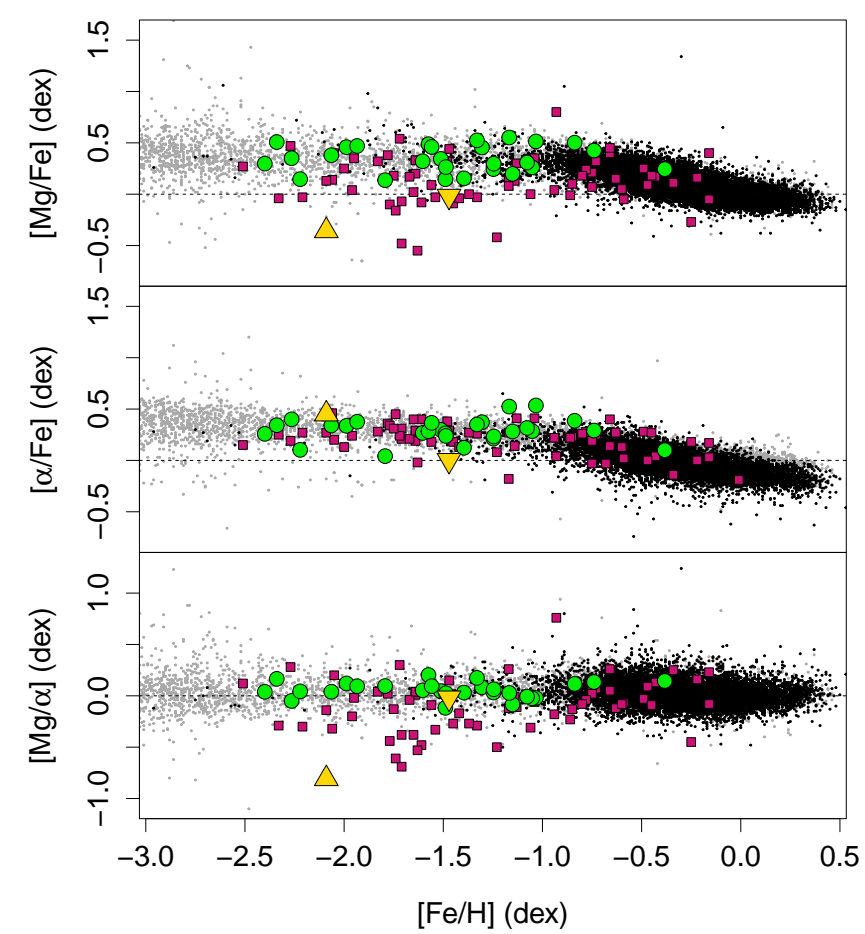

Fig. 6. Average $[\mathrm{Mg} / \mathrm{Fe}],[\alpha / \mathrm{Fe}]$, and $[\mathrm{Mg} / \alpha]$ of our collected $\mathrm{GES}$ and literature sample of $28 \mathrm{MW}$ GCs (green circles, see Sect. 4) and of the literature sample of extragalactic GCs (purple squares; see Sect. 4.3). The MW reference population is drawn from the GES iDR4 sample (black dots) and from the SAGA database of metal-poor stars (grey dots). We also plotted NGC 2419 as a yellow upward triangle and $\mathrm{Ru} 106$ as a yellow downward triangle.

field stars as $[\mathrm{Fe} / \mathrm{H}]$ increases (as in Fig. 10 by Mucciarelli et al. 2012, for the LMC). The exact distribution would be governed by the global star formation rate of each galaxy, which governs the metallicity at which the knee occurs.

Both the external and internal explanations appear viable at the moment, and they might also operate simultaneously. Further information could be obtained: (1) by obtaining large and homogeneous samples of field stars with $[\mathrm{Mg} / \alpha]$ and $[\mathrm{Fe} / \mathrm{H}]$ measurements to compare with the available GC measurements on a galaxy-by-galaxy basis; and (2) by obtaining large sample of individual star abundances for the nearest extragalactic GCs .

\section{Summary and conclusions}

We used GES iDR4 data on calibrating globular clusters to explore the $\mathrm{Mg}-\mathrm{Al}$ anti-correlation, which is well measured in the GES observing set-ups and varies significantly from one GC to the other, and therefore can provide strong constraints on the GC properties that control the anti-correlation phenomenon.

Even if iDR4 is a preliminary and intermediate data release, it was the first one in which many different loops of the internal and external calibration were closed in the complex GES homogenization workflow (see P16; Hourihane et al., in prep.; and Randich et al., in prep.). As result, the agreement between UVES and GIRAFFE is within the quoted uncertainties with $0.10-0.15$ dex median differences; there are no significant trends of abundance ratios with the APs, in particular with $T_{\text {eff }}$ or $\log g$; and there are small offsets with the high-resolution literature data of no more than 0.1 dex. We also add a new GC, NGC 5927, one 
of the most metal-rich GCs, which was included in GES to facilitate the internal calibration in conjunction with open clusters.

Given the excellent agreement with the literature, we assembled a homogenized database of $\simeq 1300$ stars in 28 GCs with $[\mathrm{Fe} / \mathrm{H}],[\mathrm{Mg} / \mathrm{Fe}]$, and $[\mathrm{Al} / \mathrm{Fe}]$ measurements from GES iDR4, the FLAMES GC survey (Carretta et al. 2009a,b, and other papers cited above), and the APOGEE survey (Mészáros et al. 2015). We explored two different open topics as a demonstration of the presented data quality. The first topic concerns the dependency of the $\mathrm{Mg}-\mathrm{Al}$ anti-correlation extension with GC global parameters. In particular, it was suggested by Carretta et al. (2009a) that the extension depends on both mass and metallicity, but no formal analysis was performed in that paper owing to the limited sample. The suspicion was supported by the Mészáros et al. (2015) data. However a different analysis by Cabrera-Ziri et al. (2016) found a very weak relation between the $\mathrm{Mg}$-Al extension and $[\mathrm{Fe} / \mathrm{H}]$ with a large spread and low statistical significance from a literature database of $20 \mathrm{GC}$ measurements. We profited from our large homogenized sample, which includes NGC 5927, and we employed a linear fit on cluster mass and metallicity simultaneously. Our analysis removes any remaining doubt about the fact the the $\mathrm{Mg}$-Al anti-correlation extension depends on both mass and metallicity. Adding age as a third parameter worsened the fit and we concluded that the $\mathrm{Mg}$ - $\mathrm{Al}$ anti-correlation does not change significantly with age.

We also explored another open topic related to the low $[\mathrm{Mg} / \alpha]$ measured in some extragalactic GCs (Larsen et al. 2014; Colucci et al. 2014; Sakari et al. 2015) to see whether a highly extended $\mathrm{Mg}-\mathrm{Al}$ anti-correlation could explain the observed trends. We made the reasonable hypothesis that an average of the available individual star abundances is comparable with the abundances obtained by integrated light spectroscopy (see Colucci et al. 2017, and references therein). We concluded that a normal anti-correlation, no matter how extended, would not reproduce those low $[\mathrm{Mg} / \alpha]$ values. A more extreme chemical composition, such as that of NGC 2419 (Mucciarelli et al. 2012; Cohen \& Kirby 2012; Carretta et al. 2013b; Ventura et al. 2012), would be required. Besides this explanation, related to the internal GC chemical properties, there is another external explanation related to the global chemical evolution properties of the host galaxy and the yields of SNe type Ia and II, but the data available so far do not allow us to discriminate between the two, which could be either mutually exclusive or coexist in different GC populations.

We conclude that the GES data have a quality sufficient to explore the presented and many other topics related to the chemistry of GCs, providing clear results. When the whole sample of GCs and of the observed stars have been analyzed, also including elements that are not completely determined in iDR4, it will be possible to statistically analyze the entire set of elements that vary in GCs.

Acknowledgements. We warmly thank I. Cabrera-Ziri for a discussion on the biases in determining the extent of the $\mathrm{Mg}$-Al anti-correlation; A. Mucciarelli for a discussion on anomalous GCs like NGC 2419; S. Larsen for a discussion on the phenomenon of low $[\mathrm{Mg} / \alpha]$ in extragalactic GCs; M. Gieles for a discussion on the possible polluters and their impact on the $\mathrm{Mg}$-Al anti-correlation extension; and the referee of this paper, P. Ventura, who offered his insights to improve the manuscript both in its substance and form. This research has made use of the following softwares, databases, and online resources: topcat (Taylor 2014); the CDS and Vizier databases (http://cdsportal.u-strasbg.fr/) the R project (https://www.r-project.org), and Rstudio (https://www. rstudio.com/). This research is based on data products from observations made with ESO Telescopes at the La Silla Paranal Observatory under programme ID 188.B-3002. These data products have been processed by the Cambridge Astronomy Survey Unit (CASU) at the Institute of Astronomy, University of
Cambridge, and by the FLAMES/UVES reduction team at INAF/Osservatorio Astrofisico di Arcetri. These data have been obtained from the Gaia-ESO Survey Data Archive, prepared and hosted by the Wide Field Astronomy Unit, Institute for Astronomy, University of Edinburgh, which is funded by the UK Science and Technology Facilities Council. This work was partly supported by the European Union FP7 programme through ERC grant number 320360 and by the Leverhulme Trust through grant RPG-2012-541. We acknowledge the support from INAF and Ministero dell' Istruzione, dell' Università e della Ricerca (MIUR) in the form of the grant "Premiale VLT 2012". The results presented here benefit from discussions held during the Gaia-ESO workshops and conferences supported by the ESF (European Science Foundation) through the GREAT Research Network Programme. E.P. and D.R. benefited from the International Space Science Institute (ISSI; Bern, CH), through funding of the International Team "The Formation and Evolution of the Galactic Halo". M.T.C. acknowledges the financial support from the Spanish Ministerio de Economía y Competitividad, through grant AYA2013-40611-P. S.G.S. acknowledges the support by Fundação para a Ciência e Tecnologia (FCT) through national funds and a research grant (project Ref. UID/FIS/04434/2013, and PTDC/FIS-AST/7073/2014). S.G.S. also acknowledges the support from FCT through Investigador FCT contract of reference IF/00028/2014 and POPH/FSE (EC) by FEDER funding through the programme "Programa Operacional de Factores de Competitividade - COMPETE". D.G., B.T., and S.V. gratefully acknowledge support from the Chilean BASAL Centro de Excelencia en Astrofísica y Tecnologías Afines (CATA) grant PFB06/2007. L.M. acknowledges support from Proyecto Interno of the Universidad Andres Bello. E.J.A was supported by Spanish MINECO under grant AYA201675931-C2-1-P with FEDER funds. R.S. acknowledges support from the Polish Ministry of Science and Higher Education (660/E-60/STYP/10/2015). Support for L.S. was provided by the Chile's Ministry of Economy, Development, and Tourism's Millennium Science Initiative through grant IC120009, awarded to The Millennium Institute of Astrophysics, MAS.

\section{References}

Bastian, N., Cabrera-Ziri, I., \& Salaris, M. 2015, MNRAS, 449, 3333 Blanco-Cuaresma, S., Soubiran, C., Jofré, P., \& Heiter, U. 2014, A\&A, 566, A98 Cabrera-Ziri, I., Lardo, C., Davies, B., et al. 2016, MNRAS, 460, 1869 Carretta, E. 2015, ApJ, 810, 148

Carretta, E., Bragaglia, A., Gratton, R., \& Lucatello, S. 2009a, A\&A, 505, 139

Carretta, E., Bragaglia, A., Gratton, R. G., et al. 2009b, A\&A, 505, 117 Carretta, E., Bragaglia, A., Gratton, R. G., et al. 2010, A\&A, 516, A55 Carretta, E., Lucatello, S., Gratton, R. G., Bragaglia, A., \& D’Orazi, V. 2011, A\&A, 533, A69

Carretta, E., Bragaglia, A., Gratton, R. G., et al. 2013a, A\&A, 557, A138 Carretta, E., Gratton, R. G., Bragaglia, A., et al. 2013b, ApJ, 769, 40 Carretta, E., Bragaglia, A., Gratton, R. G., et al. 2014, A\&A, 564, A60 Cohen, J. G., \& Kirby, E. N. 2012, ApJ, 760, 86

Colucci, J. E., Bernstein, R. A., Cameron, S., McWilliam, A., \& Cohen, J. G. 2009, ApJ, 704, 385

Colucci, J. E., Bernstein, R. A., \& Cohen, J. G. 2014, ApJ, 797, 116

Colucci, J. E., Bernstein, R. A., \& McWilliam, A. 2017, ApJ, 834, 105

Cordero, M. J., Pilachowski, C. A., Johnson, C. I., et al. 2014, ApJ, 780, 94

Decressin, T., Meynet, G., Charbonnel, C., Prantzos, N., \& Ekström, S. 2007, A\&A, 464, 1029

Dekker, H., D'Odorico, S., Kaufer, A., Delabre, B., \& Kotzlowski, H. 2000, in Optical and IR Telescope Instrumentation and Detectors, eds. M. Iye, \& A. F. Moorwood, Proc. SPIE, 4008, 534

de Laverny, P., Recio-Blanco, A., Worley, C. C., \& Plez, B. 2012, A\&A, 544, A126

de Mink, S. E., Pols, O. R., Langer, N., \& Izzard, R. G. 2009, A\&A, 507, L1 Denissenkov, P. A., VandenBerg, D. A., Hartwick, F. D. A., et al. 2015, MNRAS, 448,3314

D’Ercole, A., Vesperini, E., D’Antona, F., McMillan, S. L. W., \& Recchi, S. 2008, MNRAS, 391, 825

Gilmore, G., Randich, S., Asplund, M., et al. 2012, The Messenger, 147, 25 Gratton, R., Sneden, C., \& Carretta, E. 2004, ARA\&A, 42, 385 Gratton, R. G., Carretta, E., \& Bragaglia, A. 2012, A\&ARv, 20, 50 Grevesse, N., Asplund, M., \& Sauval, A. J. 2007, Space Sci. Rev., 130, 105 Gustafsson, B., Edvardsson, B., Eriksson, K., et al. 2008, A\&A, 486, 951 Harris, W. E. 1996, AJ, 112, 1487

Harris, W. E. 2010, ArXiv e-prints [arXiv: 1012.3224]

Hawkins, K., Jofre, P., Heiter, U., et al. 2016, A\&A, 592, A70

Heiter, U., Jofré, P., Gustafsson, B., et al. 2015a, A\&A, 582, A49

Heiter, U., Lind, K., Asplund, M., et al. 2015b, Phys. Scr., 90, 054010 Jeffries, R. D., Jackson, R. J., Cottaar, M., et al. 2014, A\&A, 563, A94 Jofré, P., Heiter, U., Soubiran, C., et al. 2014, A\&A, 564, A133 Johnson, J. A., Ivans, I. I., \& Stetson, P. B. 2006, ApJ, 640, 801 Kraft, R. P. 1994, PASP, 106, 553 
Lardo, C., Pancino, E., Bellazzini, M., et al. 2015, A\&A, 573, A115

Larsen, S. S. 2016, in The General Assembly of Galaxy Halos: Structure, Origin and Evolution, eds. A. Bragaglia, M. Arnaboldi, M. Rejkuba, \& D. Romano, IAU Symp., 317, 120

Larsen, S. S., Brodie, J. P., \& Strader, J. 2012a, A\&A, 546, A53

Larsen, S. S., Strader, J., \& Brodie, J. P. 2012b, A\&A, 544, L14

Larsen, S. S., Brodie, J. P., Forbes, D. A., \& Strader, J. 2014, A\&A, 565, A98

Larsen, S. S., Brodie, J. P., \& Strader, J. 2017, A\&A, 601, A96

Letarte, B., Hill, V., Jablonka, P., et al. 2006, A\&A, 453, 547

Mandushev, G., Staneva, A., \& Spasova, N. 1991, A\&A, 252, 94

Marín-Franch, A., Aparicio, A., Piotto, G., et al. 2009, ApJ, 694, 1498

Mateluna, R., Geisler, D., Villanova, S., et al. 2012, A\&A, 548, A82

McLaughlin, D. E., \& van der Marel, R. P. 2005, ApJS, 161, 304

Mészáros, S., Martell, S. L., Shetrone, M., et al. 2015, AJ, 149, 153

Milone, A. P., Piotto, G., Renzini, A., et al. 2017, MNRAS, 464, 3636

Modigliani, A., Mulas, G., Porceddu, I., et al. 2004, The Messenger, 118, 8

Mucciarelli, A., Carretta, E., Origlia, L., \& Ferraro, F. R. 2008, AJ, 136, 375

Mucciarelli, A., Origlia, L., Ferraro, F. R., \& Pancino, E. 2009, ApJ, 695, L134

Mucciarelli, A., Origlia, L., \& Ferraro, F. R. 2010, ApJ, 717, 277

Mucciarelli, A., Bellazzini, M., Ibata, R., et al. 2012, MNRAS, 426, 2889

Mucciarelli, A., Dalessandro, E., Ferraro, F. R., Origlia, L., \& Lanzoni, B. 2014, ApJ, 793, L6

Pancino, E., \& the Gaia-ESO Survey collaboration 2017, A\&A, 598, A5
Pasquini, L., Avila, G., Allaert, E., et al. 2000, in Optical and IR Telescope Instrumentation and Detectors, eds. M. Iye, \& A. F. Moorwood, Proc. SPIE, 4008, 129

Randich, S., Gilmore, G., \& Gaia-ESO Consortium. 2013, The Messenger, 154, 47

Renzini, A., D’Antona, F., Cassisi, S., et al. 2015, MNRAS, 454, 4197

Sacco, G. G., Morbidelli, L., Franciosini, E., et al. 2014, A\&A, 565, A113

Sakari, C. M., Venn, K. A., Mackey, D., et al. 2015, MNRAS, 448, 1314

Smiljanic, R., Korn, A. J., Bergemann, M., et al. 2014, A\&A, 570, A122

Suda, T., Katsuta, Y., Yamada, S., et al. 2008, PASJ, 60, 1159

Taylor, M. B. 2014, in Astronomical Data Analysis Software and Systems XXIII, eds. N. Manset, \& P. Forshay, ASP Conf. Ser., 485, 257

Tsujimoto, T., \& Bekki, K. 2012, ApJ, 751, L35

VandenBerg, D. A., Brogaard, K., Leaman, R., \& Casagrande, L. 2013, ApJ, 775, 134

Ventura, P., D’Antona, F., Di Criscienzo, M., et al. 2012, ApJ, 761, L30

Ventura, P., García-Hernández, D. A., Dell’Agli, F., et al. 2016, ApJ, 831, L17

Villanova, S., Geisler, D., Carraro, G., Moni Bidin, C., \& Muñoz, C. 2013, ApJ, 778,186

Vink, J. S., Muijres, L. E., Anthonisse, B., et al. 2011, A\&A, 531, A132

Yong, D., Grundahl, F., Nissen, P. E., Jensen, H. R., \& Lambert, D. L. 2005, A\&A, 438, 875

Yong, D., Roederer, I. U., Grundahl, F., et al. 2014, MNRAS, 441, 3396 